\title{
UN ARABO CIPRIOTA ROMANIZZATO? \\ Distacchi e identità fra variazione scrittoria e confessione religiosa
}

\section{Giuliano Mion}

DOI: http://dx.doi.org/10.7359/833-2017-mion

\section{INTRODUZIONE}

Nell'analisi della situazione linguistica di una comunità entra inevitabilmente in gioco il concetto di repertorio, inteso come l'insieme di lingue e/o di varietà della stessa lingua a disposizione dei parlanti. I rapporti che si instaurano tra le differenti varietà linguistiche che costituiscono il repertorio sono spesso oggetto di variazioni, ossia fenomeni di mutamento più o meno evidenti e interpretabili in senso sia diacronico sia sincronico.

Le righe seguenti sono dedicate a un particolare fenomeno di variazione, quello del repertorio scrittorio di una comunità linguistica, e, in particolare, alle ripercussioni grafiche scaturite da una condizione complessa di bilinguismo ${ }^{1}$. Più precisamente, esse prendono le mosse da una ricerca più ampia che tenta di mettere in evidenza come le dinamiche identitarie di una comunità arabofona di frontiera si ripercuotano sui fatti linguistici ${ }^{2}$.

Le comunità arabofone di frontiera, che la dialettologia araba definisce «periferiche», nel più dei casi sono isole linguistiche di confessione musulmana (sunnita o sciita, a seconda dei casi) incuneate da tempi remoti in regioni non arabofone. In quanto tali, pertanto, si configurano come

1 In diverse zone del mondo, bi- e plurilinguismo si accompagnano spesso a repertori scrittori complessi. Una messe di informazioni utili in proposito è reperibile, fra gli altri, nel volume di Sebba, Mahootian e Jonsson (2012).

2 Cf. Mion 2015 e 2017. 
il risultato di un avanzamento sbilanciato dei due processi socio-storici di arabizzazione e islamizzazione ${ }^{3}$.

Il caso preso in esame in questa sede, invece, appare molto diverso perché considera la condizione particolare di Cipro: caratterizzata da una schiacciante maggioranza cristiana ortodossa di lingua greca e dalla presenza di una comunità musulmana stanziata nella zona turca e turcofona settentrionale, l'isola ospita infatti una minoranza linguistica e confessionale i cui due elementi identificativi sono l'arabofonia e l'obbedienza alla Chiesa maronita ${ }^{4}$.

La sede storica della piccola comunità di cristiani maroniti di lingua araba è Kormakiti, un villaggio situato nel territorio della Repubblica Turca di Cipro Nord. La storia più remota dei maroniti arabo-ciprioti è avvolta nell'oscurità, ma si sa per certo che la loro presenza si spiega mediante una serie di ondate migratore provenienti dalla costa sirolibanese e risalenti a periodi diversi dell'epoca medievale.

Complice l'isolamento geografico della comunità, la lingua materna dei primi maroniti giunti sull'isola, l'arabo, si è conservata nei secoli fino al giorno d'oggi. Con l'autoglottonimo sanna (< ${ }^{*} \operatorname{ls} \bar{a} n-n a$, «la lingua nostra») ci si riferisce spesso, oggi, a questa forma di neoarabo ${ }^{5}$ parlato dalla comunità maronita, una varietà orale priva di tradizione scritta, dalla tipologia nettamente sedentaria e, al contempo, con tratti diatopicamente ibridi riferibili in parte all'area sirolibanese e in parte a quella mesopotamica, e il cui profilo complessivo appare drasticamente interferito a tutti i livelli d'analisi dal greco parlato cipriota ${ }^{6}$. Come sempre in questi casi, la comunità è contraddistinta da bilinguismo: l'arabo maronita cipriota è relegato al ruolo di lingua domestica, laddove il greco è deputato agli usi propri della sfera pubblica:

Die phonologische und syntaktische Interferenz des Griechischen ist allgegenwärtig, der Wortschatz ist so weit zusammengeschmolzen, dass eine Kom-

3 Cf. Kaye - Rosenhouse 1997 per una rapida veduta d'insieme; Procházka 2007 per una proposta di identificazione della perifericità delle varietà neoarabe; Tosco - Manfredi 2013 per gli sviluppi in pidgin e creoli.

4 Sui maroniti ciprioti, in particolare Varnava - Coureas - Elia 2009, nonché Emilianides 2011.

5 Per una rapida disamina della tipologia neoaraba, fra i riferimenti possibili si veda per esempio Singer 1982.

6 I riferimenti fondamentali a questa varietà neoaraba consistono nella grammatica descrittiva Borg 1985 e nel dizionario Borg 2004. Uno studio precedente, ma frequentemente riconosciuto come meno affidabile, è Tsiapera 1969. Riflessioni di ordine genetico/ tipologico si trovano in Jastrow 1977. 
munikation ohne ständige ad-hoc-Entlehnungen aus dem Griechischen offenbar nicht mehr praktiziert werden kann. Wir haben es nicht mehr mit einem arabischen Dialekt zu tun, sondern nur noch mit den arabischen Bestandteiler einer «mixed language». ${ }^{7}$

La concezione tradizionale della diglossia fergusoniana, pertanto, che, nella sua applicazione all'arabofonia, prevede un'opposizione fra il polo «basso» del dialetto locale e il polo «alto» della cosiddetta fușha (o arabo classico), non è applicabile a questa comunità: la fușbā, che per le comunità arabofone musulmane è una lingua sacra, è di fatto assente dal repertorio linguistico dei maroniti ciprioti e, proprio in virtù della totale mancanza di qualsiasi esposizione a questa varietà, non può costituirsi come un modello normativo di riferimento; quest'ultimo, al contrario, è rappresentato semmai dal greco, unica lingua di cultura ${ }^{8}$.

L'occupazione turca del 1974, con la conseguente divisione dell'isola in due entità statali ${ }^{9}$, ha provocato una forte dispersione della comunità maronita insieme a pesanti ripercussioni linguistiche: l'arabo cipriota maronita è ormai una lingua che corre il rischio di estinguersi rapidamente.

\section{Dall'alfabeto greco all'Alfabeto latino}

In una situazione di bilinguismo come quella dei maroniti ciprioti in cui la lingua domestica è priva di tradizione scritta, è ovvio che la comunità faccia ricorso alla scrittura greca quando, in sporadici casi, decide di registrare per iscritto la propria varietà neoaraba. Al di là dei rarissimi documenti scritti del passato che sono stati redatti in arabo cipriota ${ }^{10}$, al giorno d'oggi nella produzione scritta in sanna una svolta decisiva si ha con la rivista $O$ typos ton Maroniton (Il giornale dei Maroniti) che ha scelto di dedicare alcune

7 Jastrow 1977, 261.

8 Per il caso sotto esame, mi sembra particolarmente efficace definire il bilinguismo cipriota maronita arabo-greco secondo la dicotomia «gregario»/«veicolare» che si ritrova in Calvet 1987.

9 Per una storia dell'isola in periodo contemporaneo, si veda Mallinson 2005. Si consideri comunque che, nel momento in cui questo articolo viene redatto, la Repubblica Turca di Cipro del Nord (Kuzey Kıbrıs Türk Cumburiyeti o, più rapidamente, Kuzey Kıbrıs), autoproclamatasi nel 1983, sul piano internazionale viene riconosciuta esclusivamente dalla Turchia.

10 Lentin 2000 pubblica un breve documento di natura epistolare reperito presso l'Archivio storico della Propaganda Fide. 
delle sue pagine allarabo cipriota. Il mensile, edito a Nicosia con finanziamenti della comunità maronita, rappresenta infatti, per quanto è dato di sapere, il primo esperimento di rivitalizzazione dell'arabo cipriota. Nel triennio compreso fra il 2006 e il 2009 pubblica una rubrica contenente una serie di vere e proprie lezioni di apprendimento del sanna, redatte interamente in lingua greca, con numerosi esempi di arabo cipriota scritto in alfabeto greco, e spiegazioni grammaticali basate sul modello greco ${ }^{11}$.

Il numero 69 (ottobre 2007) consiste invece nel giro di boa della politica linguistica promossa dalla rivista, in quanto riporta una lunga intervista in greco e in traduzione inglese all'arabista maltese Alexander Borg che sprona i maroniti ciprioti a rivalutare il proprio patrimonio linguistico e culturale. L'appello è fortemente evocativo perché, pur se sotto forma di divulgazione giornalistica, l'origine del sanna viene rapidamente collocata come varietà neoaraba distaccatasi dal continente in epoca remota e sopravvissuta poi autonomamente, illustrandone la parentela genetica con altre lingue illustri quali accadico e aramaico, a dimostrazione dell'antichità della lingua e di come la comunità cipriota affondi le proprie radici ancestrali nella tradizione culturale e religiosa del Vicino Oriente.

È probabilmente da questo momento che gli arabofoni ciprioti maturano una riflessione metalinguistica che, sui numeri successivi della rivista maronita e su diversi siti Internet, si manifesta in un'attenzione vigorosa nei confronti delle vicissitudini del Cristianesimo (vicino-)orientale ${ }^{12}$. Il sanna arriva così a competere indirettamente con il neoaramico di Maaloula (Siria), poiché, in alcuni ambienti, non si tarderà a concepirlo come una miracolosa sopravvivenza dell'antica lingua di Gesù nell'isola di Cipro:

If the people of this remote village were to travel back to Jesus' time and hear him preach, they wouldn't need an interpreter to understand the Sermon on the Mount or the parable of the prodigal son. That's because they speak the same language as the Son of God. Literally. Spoken in the Middle East during Jesus' time, Aramaic is still used in everyday life by most of the 130 elderly Maronite Catholics in Kormakiti, which overlooks the Mediterranean Sea. ${ }^{13}$

11 Sulla fase grecografa del sanna in O typos ton Maroniton, si rimanda all'analisi contenuta in Mion 2017.

12 Del Zanna 2011.

13 La citazione è tratta da un articolo pubblicato sul Christian Science Monitor, reperibile all'indirizzo: http://www.csmonitor.com/2004/0129/p07s02-wome.html. 


\section{LA PROPOSTA DI ROMANIZZAZIONE}

A partire dal numero 72 (gennaio 2008), la rivista prosegue la sequenza di lezioni di apprendimento del sanna, ma inizia a servirsi dell'alfabeto latino. La scelta nasce da una proposta avanzata da Borg che consiste in una vera e propria riforma scrittoria e ortografica ${ }^{14}$. Nella sua versione modificata appositamente per il sanna, l'alfabeto latino prevede i grafemi seguenti:

$$
<\mathrm{a}, \mathrm{b}, \mathrm{c}, \dot{\mathrm{c}}, \mathrm{d}, \delta, \mathrm{e}, \mathrm{f}, \dot{\mathrm{g}}, \mathrm{x}, \mathrm{i}, \mathrm{j}, \mathrm{k}, \mathrm{l}, \mathrm{m}, \mathrm{n}, \mathrm{o}, \mathrm{p}, \mathrm{r}, \mathrm{s}, \mathrm{s}, \mathrm{t}, \theta, \mathrm{u}, \mathrm{v}, \mathrm{w}, \mathrm{y}, \mathrm{z}>
$$

Alcuni dei grafemi del sistema sono una chiara emanazione dell'ortografia maltese elaborata dalla Akkademja tal-Malti (Accademia della lingua maltese) ${ }^{15}$, come per esempio:

\begin{tabular}{ccc}
\hline MALTESE & SANNA & VALORE \\
\hline$<\dot{\mathrm{c}}>$ & $<\dot{\mathrm{c}}>$ & {$[\mathrm{t}]$} \\
$<\dot{\mathrm{g}}>$ & $<\dot{\mathrm{g}}>$ & {$[\mathrm{d}]$} \\
\hline
\end{tabular}

Altri grafemi si discostano totalmente dal modello maltese, come soprattutto il caso $\mathrm{di}<\mathrm{x}>$. In sanna, questo grafema ha un valore fricativo uvulare $[\chi]$ e deve essere tributario di una rielaborazione del simbolo IPA $[\chi]$ e/o di una confluenza con quello fricativo velare $[\mathrm{x}]$, peraltro in uso regolarmente come simbolo di trascrizione anche in dialettologia araba. In maltese, invece, lo stesso grafema ha un valore [J], cui corrisponde <ş> nel sanna, ed è originato a sua volta dalla resa grafica medievale $<x>$ con cui il castigliano rendeva la sibilante palatale dell'arabo andaluso.

Un ulteriore elemento rilevante di ingegneria linguistica è rappresentato dal grafismo <il-> atto a registrare l'articolo determinativo (cf. ar.cl. 'al). Il grafema $<$ i $>$ rende senz'altro il fonema /i/ dell'articolo, ma va pur detto che la realizzazione fonetica conosce gli allofoni (perlopiù contestuali) di $[i$, I, e]. La presenza della barra centrale <->, atta a segmentare articolo e nominale, si configura inoltre come un chiaro segno della precisione del sistema nonché della sua artificialità e ha un corrispondente diretto nella soluzione grafica con cui l'ortografia maltese segnala l'articolo.

$14 \mathrm{Si}$ veda in proposito il breve contributo di Borg, formalmente inedito, ma reperibile all'indirizzo: http://static1.1.sqspcdn.com/static/f/199216/1328511/1202758389953/ The_CMA_Alphabet.pdf?token=0IkFoJ4mvca\%2B28dkBp7xRR\%2BMd00\%3D.

15 Cf. Akkademja tal-Malti 1998. 
L'ortografia di Borg conosce una fase iniziale di discreto successo, almeno stando a quanto si evince dal progetto Xki fi Sanna (Parla la nostra lingua) promosso nel 2008 da alcuni esponenti della comunità maronita cipriota ${ }^{16}$. Il progetto mirava a sensibilizzare il pubblico locale nei confronti del sanna attraverso campagne di alfabetizzazione e campi estivi per bambini e adolescenti da svolgersi a Nicosia e dintorni. Le lezioni preparate da Koumettos Katsioloudis, insegnante della scuola primaria St Maron, piuttosto «snelle» sul piano puramente glottodidattico, si servono del sistema grafico romanizzato fin qui esposto ${ }^{17}$. Valga per tutti uno fra i tanti esempi, scelto a caso, come quello dell'enunciato:

$<$ Ismi o Pavlo u 1-ism tel 1-yapati o Mario>

ism-i o pavlo w-l-ism tel l-yapat-i o mario

«il mio nome è Paolo e il nome di mio padre è Mario» ${ }^{18}$

Questo stesso autore, attivo nella promozione del sanna, suole sponsorizzare la romanizzazione anche su rete. A tal proposito, si osservi il testo seguente trilingue (sanna, greco e inglese) che compare nella pagina del video del brano musicale Ya Lubnan (Ob Libano!') ${ }^{19}$ :

Caney kişcu modcey xtir

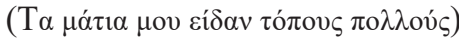

(My eyes have seen many places)

ma pşiklak ma kişcu axar,

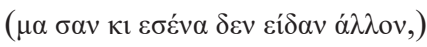

(but like you have seen no other,)

ta lu arkca mi l-Rabi xusn,

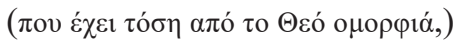

(with such godly beauty,)

16 http://sana.squarespace.com.

17 http://sana.squarespace.com/first-steps-in-cypriot-maronit/.

18 Una certa «ingenuità» di formulazione dell'enunciato si nota peraltro dalla ridondanza di /1/ che compare una prima volta in $\langle t e l\rangle$ (di fatto segmentabile in te-l-, ovvero preposizione genitivale seguita da articolo determinativo) e una seconda, subito dopo, come elemento determinativo prefisso in <l-yiapati>.

19 Interpretazione di Juliana Bou Mansour, musiche di Demetris Costantinou, testo di Koumettos Katsioloudis. Reperibile all'indirizzo: https://www.youtube.com/ watch?v=UdCnzTThnvI. 
l-softaşice, l-layl, l-saxxar.

(

(in the afternoon, at dusk, at dawn.)

L-sayf jit, ye, jit u kşacatak

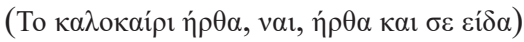

(In the summer I arrived, yes, I came to see you)

te l-kalpi savayt xatra,

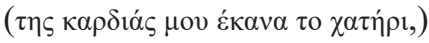

(my hearts wish I granted,)

ruxt fi l-Anaya u fi l-Beirut,

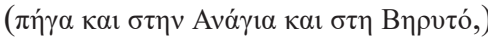

(I travelled to Anayia and to Beirut,)

$\theta$ katist fi l-Xarisa l-Catra.

(

(attended mass at the Holy Mother of Harisa.)

\section{VERSO Un'ORTOGRAFia SPONTANEA}

Il successo di una campagna di acquisition planning è dato indubbiamente anche dalla qualità dell'offerta di servizi nella lingua bersaglio. Nel caso sotto esame di $O$ typos ton Maroniton, il passaggio da una semplice rubrica che propone rudimenti di sanna a veri e propri articoli giornalistici concepiti per la fruizione di massa mostra un salto di qualità notevole che va a inquadrarsi nella campagna di acquisition planning promossa dalla rivista.

A partire dal numero 88 (ottobre 2009), infatti, O typos ton Maroniton pubblica articoli di attualità dalla lunghezza di volta in volta crescente: inizialmente due mezze colonne, in seguito pagine intere. Quando l'operazione è, almeno apparentemente, ben lanciata, la rivista modifica il titolo alla rubrica in sanna che diventa $<$ Ash xabar $>$ (Che notizie?), e le aumenta sia lo spazio a disposizione (due pagine intere) sia la qualità grafica.

L'ortografia di Borg diventa senza dubbio un punto di riferimento obbligatorio per chi intende mettere il sanna per iscritto, perché ha comunque il merito di soppiantare l'alfabeto greco come unico strumento di registrazione scritta della lingua e di imporre al suo posto l'alfabeto latino. Va tuttavia osservato che nei generi testuali originati dalla comunicazione 
mediata da computer, contraddistinti da una natura meramente spontanea, numerosi esempi documentano il superamento del sistema di Borg ${ }^{20}$. Le soluzioni apparentemente troppo maltesi o troppo distanti da uno spirito grecografo vengono talora disattese per assecondare, al loro posto, opzioni che siano tributarie esclusivamente dell'alfabeto latino e, più in particolare, delle abitudini anglografe.

In tal senso, si osservi lo schema seguente dove, per alcuni fonemi, alla colonna I corrisponde l'abitudine grecografa precedente alla proposta di Borg, alla II la romanizzazione proposta da Borg, alla III l'ortografia spontanea che si delinea come un superamento della seconda fase:

\begin{tabular}{|c|c|c|c|}
\hline I & II & III & VALORE \\
\hline$<\lambda^{\prime}>$ & $<\mathrm{il}->$ & $<\mathrm{il}->/<\mathrm{l}^{\prime}>$ & il- (art.) \\
\hline$<0 v>$ & $<$ ou $>$ & $<\mathrm{u}>$ & {$[\mathrm{u}]$} \\
\hline$<\tau \zeta>$ & $<\dot{\mathrm{g}}>$ & $<\mathrm{dj}>/<\mathrm{tj}>$ & {$\left[d_{3} \sim 3\right]$} \\
\hline$<\theta>$ & $<\theta>$ & $<$ th $>$ & {$[\theta]$} \\
\hline$<\delta>$ & $<\delta>$ & $<\mathrm{d}>$ & [ð] \\
\hline$<\operatorname{sh}>$ & $<$ Ş> & $<\operatorname{sh}>$ & {$[f]$} \\
\hline
\end{tabular}

\section{RifLESSIONI CONCLUSIVE}

Al di là della campagna di rivitalizzazione del sanna che la rivista cipriota ha inteso mettere in atto, quel che pare rilevante per un'interpretazione identitaria dei fenomeni linguistici è il cambiamento del codice scrittorio di base, ossia dell'alfabeto.

In una prima fase, il sanna appare esclusivamente in alfabeto greco, ossia nella scrittura più diffusa a Cipro.

Il passaggio all'alfabeto latino, invece, sembra corrispondere a una presa di coscienza della propria identità favorita dalle campagne di ecologia linguistica e di rivitalizzazione della propria tradizione culturale maronita. Non deve trattarsi infatti di un caso che le pagine di $O$ typos ton Maroniton dimostrino una prossimità intellettuale ed affettiva costante nei confronti del Libano, che arriva ad essere dipinto in più occasioni come la madre-

20 Sul ruolo di Internet, in particolare, come generatore di nuove tipologie testuali in lingua araba, si veda per esempio Allman 2009. 
patria della comunità maronita cipriota. Quasi tutti i numeri della rivista riportano notizie sulla politica libanese e sui rapporti fra i maroniti ciprioti e quelli libanesi, interviste a esponenti del clero maronita o artisti e cantanti maroniti provenienti dal Libano. Lo stesso apparato iconografico cui la rivista fa ampio e sapiente ricorso, con le immagini dei faraglioni della corniche di Beirut o dei cedri innevati della valle della Bekaa, non può che risultare alla lunga altamente evocativo.

$\mathrm{Ma}$ in un quadro così complesso, quel che più stupisce è il passaggio apparentemente indolore da un codice scrittorio all'altro, dal momento che gli attivisti del sanna redigono ormai abitualmente i loro testi in alfabeto latino.

E del resto un fenomeno noto e osservabile in diverse zone e culture del mondo che a una variazione linguistica corrisponda anche una variazione scrittoria ${ }^{21}$. Nei casi più esasperati, il codice scrittorio può mutare drasticamente per via di un sentimento di dissociazione dalla cultura maggioritaria o, perlomeno, di avvicinamento a un'altra cultura ritenuta più prossima alla propria. Il caso di alcune delle ex repubbliche sovietiche che, nel corso di qualche decina di anni, hanno vissuto più di un mutamento scrittorio, rimane senza dubbio fra i più emblematici ${ }^{22}$.

In arabofonia, un caso recente sicuramente curioso è il tentativo di alcuni linguisti sauditi di modificare l'alfabeto arabo al fine di sostituirlo completamente all'IPA, un codice altamente tecnico che viene percepito come sistema eccessivamente eurocentrico ${ }^{23}$. Ma d'altronde la scrittura nei paesi arabi ha dato luogo a un dibattito di lunga data che ha sempre visto schierarsi fazioni diametralmente opposte di riformatori, promotori di processi di semplificazione funzionale dell'alfabeto arabo, e di conservatori, legati perlopiù all'ideologia dell'immutabilità della lingua sacra ${ }^{24}$.

Escludendo la rivoluzione linguistica turca (il dil devrimi che nel 1928 ha consentito il passaggio da osmanlı a turco moderno), occorrerà conclu-

21 La letteratura in proposito è vastissima e, per una prima presa di contatto con la tematica, si rimanda in prima istanza a Cardona 1981, Coulmas 2013 e Mancini - Turchetta 2014. Sul problema più specifico dell'elaborazione ortografica, che rappresenta una seconda fase dopo quella della scelta del codice scrittorio, si vedano Sebba 2007, Dal Negro - Guerini - Iannàccaro 2015, nonché la sintesi metodologica di Rutkowska - Rössler 2014.

22 Il tagiko, per esempio, in pochi anni è passato dall'alfabeto arabo a quello latino, e da quest'ultimo al cirillico.

23 Mion 2014.

24 Meynet 1971, per esempio, ricorda come delle oltre trecento proposte di riforma scrittoria avanzate dal 1938 al 1968 all'Accademia di Lingua Araba del Cairo nessuna ha avuto un seguito. 
dere che in ambiente arabofono gli unici distacchi concreti rispetto alla tradizione sono costituiti da due casi che, pur geograficamente distanti fra di loro, sono accomunati da un'identità confessionale condivisa: il caso del maltese e quello dell'arabo libanese della comunità cristiana.

Il primo, sebbene si configuri storicamente come una varietà neoaraba maghrebina, è parlato da una popolazione che ha fatto del Cristianesimo, e più in particolare del Cattolicesimo, la sua unica professione di fede. La dissociazione dalla cultura arabo-musulmana, già facilitata dal fattore confessionale, è passata definitivamente anche attraverso la scelta di un codice scrittorio che rimanda ideologicamente all'ambiente cristiano, malgrado la discutibile economia di certe soluzioni grafiche rispetto al sistema fonologico della lingua ${ }^{25}$. La riforma linguistica e il riconoscimento del maltese, infine, hanno avuto una sua ultima fase decisiva a ridosso del secondo conflitto mondiale, quando peraltro l'allontanamento del maltese dalla sfera d'influenza della cultura italiana è stata favorita dagli inglesi ${ }^{26}$.

Il secondo caso riguarda la proposta del libanese cristiano maronita Saïd Aql (1912-2014) il quale, sulla scia della corrente ideologica «fenicista» che respingeva qualsiasi legame tra il Libano e la cultura arabo-islamica ma rilanciava l'eredità culturale fenicia del paese, negli anni Sessanta ha proposto l'adozione dell'alfabeto latino ${ }^{27}$. Mentre il tentativo maltese si è concluso con successo, la proposta libanese non ha sortito effetti sostanziali, probabilmente per via dell'elevatissimo livello di rielaborazione e di artificialità delle modifiche proposte che, anziché facilitarne l'apprendimento da parte dei cristiani libanesi, normalmente bilingui con il francese, finisce paradossalmente per scoraggiarne l'adozione ${ }^{28}$.

In conclusione, il mutamento scrittorio che ha investito il sanna in pochi anni non sembra sottrarsi alla tendenza universale che fa della confessione religiosa il motore delle scelte relative alle pratiche scrittorie di una comunità. Il caso maltese e quello cristiano libanese favoriscono una dissociazione dal retaggio spiccatamente arabo che viene inevitabilmente accostato all'Islam. L'esempio del sanna, invece, ricorda maggiormente quanto accade in Europa orientale dove la scelta del codice scrittorio (alfabeto cirillico o alfabeto latino) demarca immancabilmente l'aderenza alla Chiesa cat-

25 Si pensi a soluzioni grafiche come $<\hbar>$, pur calcato sull'IPA, o ancora più significativamente del polifunzionale $<$ gh>.

26 Cf. Vanhove 2008. Per una storia del maltese, Brincat 2000.

27 Cf. Płonka 2006.

28 È evidente che soluzioni più allineate alle tradizioni ortografiche di lingue di grande cultura, come l'inglese o il francese, o al limite anche più vicine al sistema maltese, di per sé già poco economico, avrebbero sicuramente aiutato la causa di Saïd Aql. 
tolica o a quella ortodossa. Nel caso cipriota, in particolare, la scelta della scrittura finisce per rappresentare una dissociazione dalla Chiesa ortodossa e confermare così ancora una volta il legame tra la Chiesa cattolica e quella maronita, unica Chiesa d'Oriente, con sede in Libano, ad essere rimasta nei secoli fedele a Roma.

\section{RIFERIMENTI BIBLIOGRAFICI}

Akkademja tal-Malti 1998

Allman 2009

Borg 1985

Borg 2004

Brincat 2000

Calvet 1987

Cardona 1981

Coulmas 2013

Dal Negro - Guerini Iannàccaro 2015

Del Zanna 2011

Emilianides 2011
Akkademja tal-Malti, Regoli tal-kitba tal-Malti, Valletta, Klabb Kotba Maltin, 1998.

K. Allman, «Arabic Language Use Online: Social, Political, and Technological Dimensions of Multilingual Internet Communication», The Monitor: Journal of International Studies 15 (2009), 61-76.

A. Borg, Cypriot Arabic: A Historical and Comparative Investigation into the Phonology and Morphology of the Arabic Vernacular Spoken by the Maronites of Kormakiti Village in the Kyrenia District of North-Western Cyprus, Stuttgart, Deutsche Morgenländische Gesellschaft, 1985.

A. Borg, A Comparative Glossary of Cypriot Maronite Arabic (Arabic-English), Leiden, Brill, 2004.

J. Brincat, Il-Malti. Elf sena ta' storja, Malta, PIN, 2000 .

L.-J. Calvet, La guerre des langues et les politiques linguistiques, Paris, Payot, 1987.

G.R. Cardona, Antropologia della scrittura, Torino, Loescher, 1981.

F. Coulmas, Writing and Society: An Introduction, Cambridge, Cambridge University Press, 2013.

S. Dal Negro - F. Guerini - G. Iannàccaro (a cura di), Elaborazione ortografica delle varietà non standard. Esperienze spontanee in Italia e all'estero, Bergamo, Sestante, 2015.

G. Del Zanna, I Cristiani e il Medio Oriente (17981924), Bologna, il Mulino, 2011.

A.C. Emilianides, Religion and Law in Cyprus, Alphen aan den Rijn, Kluwer Law International, 2011. 
Jastrow 1977

Kaye - Rosenhouse 1997

Lentin 2000

Mallinson 2005

Mancini - Turchetta 2014

Meynet 1971

Mion 2014

Mion 2015

Mion 2017

Płonka 2006

Procházka 2007

Rutkowska - Rössler 2014
O. Jastrow, «Gedanken zum zypriotischen Arabisch», Zeitscbrift der Deutschen Morgenländischen Gesellschaft 127 (1977), 258-286.

A.S. Kaye - J. Rosenhouse, «Arabic Dialects and Maltese», in R. Hetzron (ed.), The Semitic Languages, New York, Routledge, 1997, 263-311.

J. Lentin. «A 17th Century Document in Cypriot Arabic», in M. Mifsud (ed.), Proceedings of the Third International Conference of Aida, Malta, Salesian Press, 2000, 207-211.

W. Mallinson, Cyprus: A Modern History, London, I.B. Tauris, 2005.

M. Mancini - B. Turchetta, Etnografia della scrittura, Roma, Carocci, 2014.

R. Meynet, L'écriture arabe en question. Les projets de l'Académie de Langue Arabe du Caire de 1938 à 1968. Beyrouth, Dar el-Machreq, 1971.

G. Mion, «Arabiser la phonétique. L'arabisation de l'Alphabet Phonetique International», Acta Orientalia Academiae Scientiarum Hungaricae 67 (2014), 289303.

G. Mion, «Sull'interferenza fonologica in arabo cipriota», in C. Consani (a cura di), Contatto interlinguistico fra presente e passato, Milano, LED, 2015.

G. Mion, «Cypriot Arabic between Orality and Literacy in $O$ typos ton Maroniton», in P. Molinelli (ed.), Language and Identity in Multilingual Mediterranean Settings: Challenges for Historical Sociolinguistics, Berlin, Mouton de Gruyter, 2017, 325-339.

A. Płonka, «Le nationalisme linguistique au Liban autour de Sa'ìd 'Aql et l'idée de langue libanaise dans la revue Lebnaan en nouvel alphabet», Arabica 53 (2006), 423-471.

S. Procházka, «Does Geographical Periphery Imply Linguistic Periphery? The Examples of the Arabic Dialects of Cilicia and Urfa in Southern Turkey», Romano-Arabica 6-7 (2007), 109-132.

H. Rutkowska - P. Rössler, «Orthographic Variables», in J.M. Hernández-Campoy - J.C. Conde-Silvestre (eds.), The Handbook of Historical Sociolinguistics, New York, Wiley-Blackwell, 2014, 213-236. 
Sebba 2007

Sebba - Mahootian Jonsson 2012

Singer 1982

Tsiapera 1969

Vanhove 2008

Varnava - Coureas - Elia 2009
M. Sebba, Spelling and Society: The Culture and Politics of Orthography around the World, Cambridge, Cambridge University Press, 2007.

M. Sebba - S. Mahootian - C. Jonsson (eds.), Language Mixing and Code-switching in Writing: Approaches to Mixed-language Written Discourse, New York, Routledge, 2012, 255-272.

H.R. Singer, «Der neuarabische Sprachraum», Grundriss der arabischen Pbilologie 1 (1982), 110-118.

M. Tsiapera, A Descriptive Analysis of Cypriot Maronite Arabic, The Hague, Mouton de Gruyter, 1969.

M. Vanhove, «Lengua maltesa», in F. Corriente A. Vicente (eds.), Manual de dialectología neoárabe, Zaragoza, Ieiop, 2008, 379-407.

A. Varnava - N. Coureas - M. Elia (eds.), The Minorities of Cyprus: Development Patterns and the Identity of the Internal-exclusion, Cambridge, Cambridge Scholars, 2009. 
